\title{
The sinusitis-infertility syndrome: Young's saint, old devil
}

\section{To the Editors:}

Young's syndrome (YS) is a triad of bronchiectasis, chronic rhinosinusitis and infertility due to vas deferens obstruction, in which case cystic fibrosis (CF) and primary ciliary diskinesia are excluded. One assumed aetiology of YS is mercury exposure during childhood. During the 20th Century, mercury use was discouraged, thus reducing exposure. HENDRY et al. [1] postulated that this was the reason for the declining prevalence of YS. They observed a rate of $50 \%$ of YS in males with obstructive azoospermia who were born before 1955; however, this number plummeted to $17 \%$ in males born after 1955 . So, if mercury poisoning is truly the aetiology, it is likely that YS is declining. Furthermore, there seems to be a lack of convincing evidence describing the sinusitis component of YS, raising doubt as to whether the syndrome is a real cause of chronic sinusitis [2]. Nevertheless, YS is still regarded as a cause of bronchiectasis. In adult bronchiectatic populations, its presence is estimated to be $\sim 3 \%[3,4]$. Conversely, YS was not found in our group of 342 patients with bronchiectasis. 72 patients had non-CF bronchiectasis without YS. In the remaining $270 \mathrm{CF}$ patients, 137 were male of which 10 had one missing genotype. Of these 10 patients, only three had a negative sweat test. They all had chronic rhinosinusitis. In these patients, genetic testing discovered heterozygosity for dF508. The first patient had no obstruction of the vas deferens but an atresia, being more suggestive for CF-like disease. The second patient showed exocrine pancreatic insufficiency, diabetes, distal intestinal obstruction syndrome and a sibling with CF. Finally, the third patient had exocrine pancreatic insufficiency and diabetes. Full sequencing of the CF gene did not show a second mutation in these patients. The clinical phenotype suggested a diagnosis of CF-like disease rather than YS in these patients. The inability to detect deep intronic mutations with complete $\mathrm{CF}$ transmembrane conductance regulator (CFTR) gene array and the possibility of changed expression in co-modulatory genes makes it difficult to exclude CF genotype completely. In conclusion, we believe that YS, when caused by mercury exposure, is declining. In all other cases, YS should probably be regarded as a CF-like disease and implies further investigation by more extensive screening of the CFTR gene, which may reveal CFTR gene disease, including the milder regions of the disease spectrum [5]. Thus, once considered an old saint, YS has now become, thanks to scientific progress, truly an old devil.

\section{P.C. Goeminne and L.J. Dupont}

Respiratory Medicine, University of Leuven, Leuven, Belgium.

Correspondence: P.C. Goeminne, Respiratory Medicine, University of Leuven, Herestraat 49, 3000 Leuven, Belgium. E-mail: pieter.goeminne@student.kuleuven.be

Statement of Interest: None declared.

\section{REFERENCES}

1 Hendry WF, A'Hern RP, Cole PJ. Was Young's syndrome caused by exposure to mercury in childhood? BMJ 1993; 307: 1579-1582.

2 Arya AK, Beer HL, Benton J, et al. Does Young's syndrome exist? J Laryngol Otol 2009; 123: 477-481.

3 Pasteur MC, Helliwell SM, Houghton SJ, et al. An investigation into causative factors in patients with bronchiectasis. Am J Respir Crit Care Med 2000; 162: 1277-1284.

4 Shoemark A, Ozerovitch L, Wilson R. Aetiology in adult patients with bronchiectasis. Respir Med 2007; 101: 1163-1170.

5 Wellesley D, Schwarz M. Cystic fibrosis, Young's syndrome, and normal sweat chloride. Lancet 1998; 352: 38.

DOI: $10.1183 / 09031936.00163809$ 